\title{
A new promising treatment in systemic sclerosis: 5-fluorouracil
}

\author{
JORGE A CASAS, ${ }^{1}$ CARLOS P SUBAUSTE, ${ }^{1}$ AND \\ GRACIELA S ALARCÓN ${ }^{12}$ \\ From the 'Department of Medicine, Universidad Peruana Cayetano Heredia, Lima, Peru; and the \\ ${ }^{2}$ Department of Medicine, University of Alabama at Birmingham, Birmingham, AL, USA
}

SUMmARY Twelve patients with systemic sclerosis according to American Rheumatism Association criteria were treated with intravenous 5-fluorouracil. Significant subjective and objective improvement occurred initially in the skin and subsequently in the involved viscera and vasculature. These preliminary results suggest that 5-fluorouracil may be an effective treatment for systemic sclerosis.

Systemic sclerosis is a disease of unknown aetiology, characterised by induration and thickening of the skin. It is frequently associated with Raynaud's phenomenon and organ-system involvement including the gastrointestinal tract, lungs, heart, and kidneys. ${ }^{1}$ There are an estimated 2-12 new cases/ million/year, which is approximately 30000 new cases a year world wide. The natural course of the disease varies; complete remission occurs infrequently. Organ-system involvement is responsible for significant morbidity and mortality. A cumulative survival of $65 \%$ at two years and $35 \%$ at seven years from disease onset has been reported in one study, whereas, in another, $20 \%$ of affected individuals were alive 10 years after disease onset. ${ }^{23}$

More than $\mathbf{5 0}$ drugs have been tried as treatment for this disease. ${ }^{4-6}$ These drugs included nonsteroidals, sex and adrenal corticosteroids, immunosuppressors, antifibrotics, and others. Most of these agents have proved to be non-efficacious, with the possible exception of D-penicillamine, which may produce skin improvement and halt the progression of visceral involvement. These changes are relatively slow to appear, however, and the drug has definite and important side effects. ${ }^{4}$

The observations by one of us (CPS) of the efficacy of topically applied 5-fluorouracil (5-FU) in the treatment of a patient with plantar fibrosclerosis, and of improvement of skin manifestations in another patient with scleroderma after treatment with combination chemotherapy (methotrexate,

Accepted for publication 13 April 1987.

Correspondence to Dr Graciela S Alarcón, 419B-SRC, University of Alabama at Birmingham, Birmingham, Alabama 35294, USA. cyclophosphamide, and 5-FU) for breast cancer, prompted us to try this drug parenterally in one patient who presented with diffuse and rapidly progressive scleroderma. This patient responded dramatically to this treatment regimen and represents the first of the 12 cases presented in this report.

\section{Patients and methods}

The study was conducted in 12 patients with progressive systemic sclerosis (scleroderma) at the Arzobispo Loayza Hospital in Lima, Peru. All patients were female and either postmenopausal or using effective contraceptive methods at the time of the study. Written informed consent was obtained before participation.

Clinical evaluation was conducted by one of us (JAC) and included assessment of the skin and musculoskeletal systems as follows: $(a)$ total skin score (TSS) as developed by Medsger et al (maximum score 104$)^{8}$; (b) maximal oral opening, in $\mathrm{mm}$; (c) flexion index (distance between the third proximal interphalangeal joint and the distal palmar crease with the hand in full flexion, in $\mathrm{mm}) ;(d)$ extension index (distance between the third fingertip and the distal palmar crease with the hand in full extension, in $\mathrm{mm}$ ); (e) palmar impression (or hand print; obtained in the same manner as a finger print it objectively demonstrates an improvement in hand extension: the palmar impression becomes larger); $(f)$ range of motion of affected joints (most commonly elbows and wrists); and ( $g$ ) functional index as developed by Guillevin and Ortonne ${ }^{5}$ (which evaluates 11 activities of daily living likely to be 
affected in patients with scleroderma) (see Appendix).

In addition to a complete history and physical examination, evaluation for vascular and visceral involvement included the following tests done serially: chest radiographs and pulmonary function testing; urine analysis, 24 hour urine for protein and creatinine clearance; electrocardiograms (ECG) and MUGA scans and barium swallows. Vascular and visceral involvement (including Raynaud's phenomenon, oesophageal, pulmonary, cardiovascular, renal, and muscular involvement) were recorded as either mild, moderate, or severe and given numerical values $(1,2$, or 3$)$ for computation purposes at both baseline and follow up visits (refer to Table 1 for definitions of mild, moderate, and severe).

The patients were treated intravenously (IV) with $12.5 \mathrm{mg} / \mathrm{kg} /$ day of $5-\mathrm{FU}$ for four to five doses, followed by four additional doses $(8-10 \mathrm{mg} / \mathrm{kg}$ ) given IV every two days (inductive phase). This was followed by a weekly dose of $10-20 \mathrm{mg} / \mathrm{kg}$ IV (maintenance dose). Therapy was monitored with a complete blood count and a clinical evaluation before each dose. While treated with 5-FU our patients did not receive other pharmacological agents, such as $\mathrm{H} 2$ blockers, corticosteroids, calcium channel blockers, and/or cytotoxics, which could have conceivably influenced the course of their illness.

Measurements performed at different times during the treatment were compared using nonparametric statistical tests. A $p$ value $\leqslant 0.05$ was chosen as indicative of statistical significance.

\section{Results}

The demographic and clinical characteristics (af? baseline and last visit) of the 12 patients studied are listed in Table 2. To better illustrate our report the course of the first three patients is presented here iff some detail. Duration of treatment ranged from 1. to 20 months. Nine patients received $5-\mathrm{FU}$ for a least six months.

\section{PATIENT NO 1}

A 28 year old woman presented with severe dyspha gia, an $8 \mathrm{~kg}$ weight loss, Raynaud's phenomenon diffuse scleroderma, and severe constipation (unre sponsive to laxatives) of five months' duration? Shortly after initiating 5-FU she noticed decreased induration of her skin which was objectively shownby a decrease in the TSS (from 49 at baseline to 30 three months later). By the fourth month het constipation, dysphagia, and Raynaud's pheo nomenon had resolved. One year after initiating this treatment she discontinued the 5-FU for financiab reasons. Her constipation recurred. She resume of treatment with 5-FU and has remained asymptomatic. She has regained weight and is receiving maintenance treatment with 5-FU as described.

\section{PATIENT NO 2}

A 24 year old woman presented with diffựjes scleroderma of seven months' duration and sig nificant pulmonary involvement characterised byo progressive dyspnoea on exertion. She alsoo had decreased range of motion (owing to skin

Table 1 Scoring system for vascular and visceral involvement in patients studied

\begin{tabular}{|c|c|c|}
\hline & Mild (1) & Severe (3) \\
\hline Raynaud's & Less than five times/day & $\begin{array}{l}\text { More than } 15 \text { times/day or digital ulcerations, } \\
\text { or both }\end{array}$ \\
\hline Oesophagus & $\begin{array}{l}\text { Dysphagia to some solid foods. Normal } \\
\text { barium swallow }\end{array}$ & $\begin{array}{l}\text { Dysphagia to solid and soft foods and weight los } \\
\text { ( }>10 \% \text { pre-illness weight). Abnormal barium } \\
\text { swallow with dilatation of the lower two thirds } \\
\text { the oesophagus }\end{array}$ \\
\hline Lung & $\begin{array}{l}\text { No symptoms: vital capacity }>70 \% \text { predicted } \\
\text { and } \mathrm{CO} \text { diffusing capacity between } 50 \text { and } \\
75 \% \text { of predicted, } \mathrm{Po}_{2} \geqslant 80 \mathrm{mmHg}\end{array}$ & $\begin{array}{l}\text { Dyspnoea plus vital capacity }<50 \% \text { of predicted o } \\
\mathrm{CO} \text { diffusing capacity }<33 \% \text { of predicted, } \\
\mathrm{Po}_{2}<69 \mathrm{mmHg}\end{array}$ \\
\hline Heart & Non-specific ST-T changes & $\begin{array}{l}\text { Angina, definite ischaemic changes by } \mathrm{ECG}^{*} \text {, } \\
\text { hypokinesis by MUGA scan or an ejection } \\
\text { fraction }<30 \%\end{array}$ \\
\hline Muscle & Mild $\mathrm{EMG}^{*}$ or $\mathrm{CK}^{*}$ abnormalities & $\begin{array}{l}\text { Definite myositis clinically, biochemically, by } \\
\text { EMG or by muscle biopsy }\end{array}$ \\
\hline Renal & $\begin{array}{l}\text { Mild hypertension, or a serum creatinine } 1.5 \times \\
\text { normal, or a creatinine clearance }>80 \% \text { or a } \\
24 \text { hour protein of }<500 \mathrm{mg}\end{array}$ & $\begin{array}{l}\text { Refractory hypertension, or a serum creatinine } \\
4 \times \text { normal, or a creatinine clearance }<20 \% \text {, or } \\
24 \text { hour protein }>3 \mathrm{~g}\end{array}$ \\
\hline \multicolumn{3}{|c|}{ 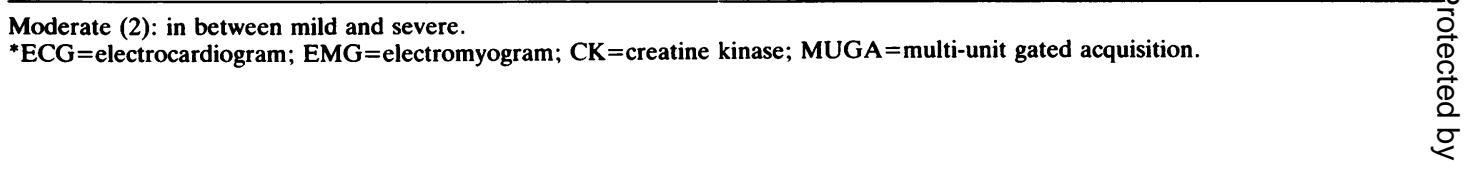 } \\
\hline
\end{tabular}


Treatment of systemic sclerosis with 5-fluorouracil 765

involvement) of the neck, shoulders, and elbows. Four weeks after the onset of treatment with 5-FU there was marked improvement in her skin and improved range of motion of the affected joints. By the fourth month of treatment her shortness of breath had subsided, and her $\mathrm{CO}$ diffusing capacity and vital capacity had improved from 30 to $82 \%$ and from 78 to $89 \%$ of predicted respectively. Fig. 1 depicts her clinical course.

PATIENT NO 3

A 42 year old woman with diffuse scleroderma of 18 months' duration presented with marked skin involvement, severe dysphagia, interstitial pulmonary disease, angina, an abnormal ECG, areas of hypokinesis by MUGA scan, and an ejection fraction of $15 \%$. She also had EMG changes diagnostic of myopathy. One month after starting 5-FU she experienced decreased induration of her skin and improved range of motion of the affected joints. Unfortunately, she developed aspiration pneumonia complicated by an empyema and, despite supportive therapy, died of cardiorespiratory failure five weeks after the initiation of 5-FU. While in hospital her white blood cell count and differential remained within normal limits.

PATIENTS $4-12$

The remaining nine patients enrolled in the study showed similar patterns of response to this treatment. By the end of the first or second week they noticed decreased skin thickening and during the subsequent four to six weeks there were objective changes shown by a decrease in the TSS (mean 36.5

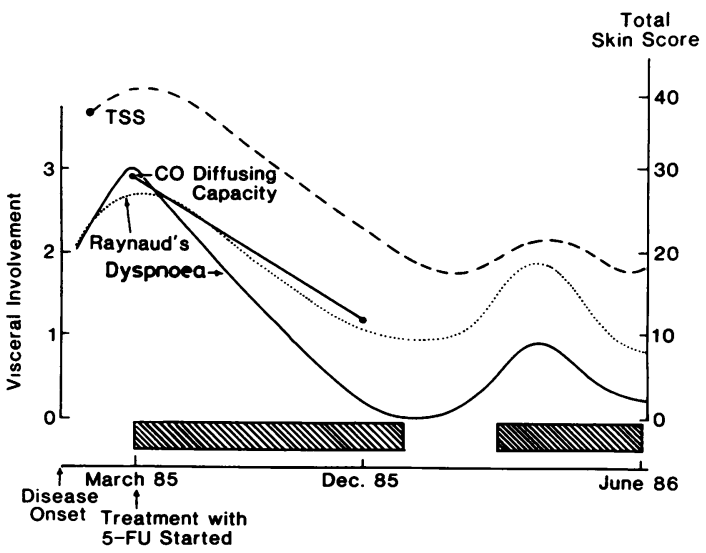

Fig. 1 Clinical course of patient No2. Observe the improvement in total skin score (TSS), Raynaud's disease, dyspnoea, and $\mathrm{CO}$ diffusing capacity as 5-FU is administered. 
at baseline; 22.0 at last visit; $p=0.01$ ), an increase in the oral opening (mean $31.3 \mathrm{~mm}$ at baseline; 33.5 at last visit; $\mathrm{p}=\mathrm{NS})$, and improvement in the flexion index (mean $98.5 \mathrm{~mm}$ at baseline; 92.6 at follow up; $\mathrm{p}=\mathrm{NS}$ ) and extension index (mean $140.1 \mathrm{~mm}$ at baseline; 146.4 at follow up; $p=N S$ ). By week 8-10 there was definite improvement of their Raynaud's phenomenon. The mean Raynaud's score was 2.2 at baseline compared with 1.3 at the last visit $(p=0.035)$. Two patients had healing of digital ulcers. The mean oesophageal score was 1.6 at baseline compared with 0.8 at the last visit $(p=0.07)$. The mean pulmonary score was 1.8 at baseline compared with 1.2 at the last visit $(p=0 \cdot 10)$. The mean functional index was 12.4 at baseline and 6.5 at the last visit $(p=0.026)$. In general, the response appeared to be related to the severity of the disease and its duration, i.e., the worse the symptoms and the shorter the duration, the more beneficial the treatment.

Of the 487 doses that our 12 patients were supposed to receive, 77 were not administered; 12 doses could not be given owing to transient unavailability of 5-FU, and the remaining 65 owing to the patients' inability to come to the hospital. It should be noted that seven of the 12 patients continue to receive 5-FU. One patient died (patient No 3, described in detail: patient died of aspiration pneumonia), and the remaining four were lost to follow up 3, 6, 11, and 15 months after starting 5-FU (patients Nos 7, 9, 4 and 2 respectively). 5Fluorouracil was discontinued temporarily in almost all patients (one or two doses). Two patients (Nos 7 and 4) stopped 5-FU for a few weeks. This was followed by reappearance or worsening of the skin lesions in patient No 7, and in patient No 4 by reappearance of digital ulcerations.

\section{SIDE EFFECTS}

In general, patient tolerance of 5-FU was very good. Side effects included mild upper gastrointestinal symptoms in seven patients, which were self limited or resolved by a change in dosing from IV bolus to IV infusion or a dose reduction of $25 \%$, or both. Two patients developed mild transient leucopenia at the end of the inductive phase of treatment that resolved when the interval of administration was increased. One patient experienced an intense headache during the infusion of his 17th dose, however this did not recur with subsequent doses.

\section{Discussion}

It is very difficult to draw conclusions about the benefit of any drug treatment in scleroderma given the variable course of this disease. ${ }^{12}$ Additionally, this study was not carried out blind and was uncontrolled. Our scoring system for vascular and $\frac{D}{\omega}$. visceral involvement uses subjective and objective criteria; these criteria were chosen not only because $\overrightarrow{\vec{A}}$ of their availability in the clinical setting where our $\frac{7}{0}$ patients were studied, but also because of their $\frac{C}{0}$ reliability and relative clinical importance in this $\overline{\bar{c}}$ disorder as noted by other investigators. ${ }^{9}$ We failed, $\vec{\nabla}$ however, to obtain serial skin biopsy specimens, which could have helped in the objective evaluation os of skin involvement. A consistent pattern of re- $\vec{O}$ sponse to therapy with 5-FU was seen in these $12 \overrightarrow{\vec{H}}$ patients and suggests a causal relation between $\vec{\sigma}$ improvement and treatment. Even those patients with the most severe form of the disease responded. ? Improvement was noted early, and began with, but $\vec{c}$ was not limited to the skin, as it included vascular $\vec{\circ}$ and visceral organs as well. When the treatment was $-v$ interrupted for whatever reason, the skin changes $\mathscr{\mathcal { E }}$ (including digital ulcerations) recurred. These 음 changes responded to reinstitution of therapy with 5-FU and were not felt to be related to seasonal 0 temperature changes. We do not yet know for how long 5-FU should be administered (at maintenance dose), but six months appear to be reasonable before considering the trial a failure; if a patie $\vec{\mathscr{}}$ continues to improve, however, a longer trial coufit. be justified. Because systemic sclerosis remains difficult disease to treat effectively we believe tha our experience, although preliminary, deserves reporting.

If proved effective, the mechanism of action of

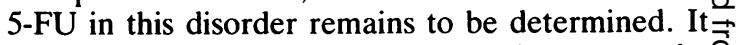
can be speculated that 5-FU could prevent the $\frac{\text { O }}{3}$ differentiation of endothelial and periendothelial cells into fibroblast and thus the active deposition of collagen. ${ }^{10}$ It is conceivable that other mechanism(s) could be operative as patients with more severe disease of shorter duration appear to do better, buto even patients with longstanding disease may show some improvement.

Because of these preliminary data we are conduct ing a double blind study in patients with newlyo diagnosed scleroderma at both collaborating institu tions.

To Dr Juan Villareal and Elizabeth Escudero (Hospital Arzobispo Loayza, Lima, Peru), Dr Anton Wille (Lichtenstein), and DrN Anthony Saway (UAB, Birmingham, USA) for their help with different aspects of this project and to Wanda Williamson forc manuscript preparation.

\footnotetext{
Appendix Functional index 5

Score:

0: without any difficulty,

1: with some difficulty,

2: with great deal of difficulty,

3: unable to do.
} 
Activities:

1. Pick up a bottle with one hand.

2. Lift a glass from the table to your mouth.

3. Turn a door knob.

4. Turn a key in keyhole.

5. Open a drawer.

6. Cut your food (using fork and knife).

7. Spread butter on your bread.

8. Hold a teaspoon.

9. Chew your food.

10. Wind your watch.

11. Write.

\section{References}

1 LeRoy E C. Scleroderma. In: Kelly W N, Harris E D, Ruddy S, Sledge C B, eds. Textbook of rheumatology. 2nd ed. Philadelphia: Saunders, 1985: 1183-205.

2 Weinberger A B. Scleroderma (systemic sclerosis): Part I. Clin Rheum Pract 1984; 2: 54-68.

3 Gilliland B C, Mannik M. Progressive systemic sclerosis (diffuse scleroderma). In: Petersdorf R G, Adams R D, Brownwald $\mathrm{E}$, Isselbacher $\mathrm{K} \mathrm{J}$, Martin $\mathrm{J} \mathrm{B}$, Wilson $\mathrm{J} \mathrm{D}$, eds.
Harrison's principles of internal medicine. 10th ed. New York: McGraw Hill, 1983: 2002-6.

4 Steen V D, Medsger T A Jr, Rodnan G P. D-Penicillamine therapy in progressive systemic sclerosis. Ann Intern Med 1982; 97: 652-9.

5 Guillevin L, Ortonne J P. Traitement de la sclérodermie. Ann Med Interne (Paris) 1983; 134: 754-65.

6 Furst D E, Hillis S, Lachenbruch P A, Paulus H E, Miller B E, Clements P J. Preliminary results of a double-blind, parallel, randomized trial of chlorambucil vs placebo in progressive systemic sclerosis (PSS). Arthritis Rheum 1986; 29: S29.

7 Steen V D, Blair S, Medsger T A. The toxicity of Dpenicillamine in systemic sclerosis. Ann Intern Med 1986; 104: 699-705.

8 Medsger T A Jr, Steen V D, Ziegler G, Rodnan G P. The natural history of skin involvement in progressive systemic sclerosis. Arthritis Rheum 1980; 23: 720.

9 Furst D E, Clements P J, Saab M, Sterz M G, Paulus H E. Clinical and serological comparisons of 17 chronic progressive systemic sclerosis (PSS) and 17 CREST syndrome patients matched for sex, age, and disease duration. Ann Rheum Dis 1984; 43: 794-801.

10 Fleischmeyer R, Perlish S, Shaw K V, Pirozzi D J. Skin capillary changes in early systemic scleroderma. Arch Dermatol 1976; 112: 1553-7. 